\title{
Effect of tobacco smoke exposure on rat tracheal submucosal glands: an ultrastructural study
}

\author{
DJ LEWIS, PR JAKINS
}

From the Department of Pathology, Huntingdon Research Centre, Huntingdon, Cambridgeshire

ABSTRACT The ultrastructural appearance of rat tracheal submucosal glands after exposure to tobacco smoke for up to two years is described. Within the mucous cells many of the rough endo- N plasmic reticulum cisternae were grossly dilated with an accumulation of amorphous, electronlucent material. The Golgi zones were prominent, and the secretion granules often contained dense cores, and appeared to have coalesced. Histochemically, increased amounts of sulphated mucus were present in exposed rats. Serous, ciliated, and myoepithelial cells were unaffected by smoke exposure.

Exposure of rats to irritants, by inhalation, produces changes in the mucus-secreting submucosal glands of the airways. Sulphur dioxide exposure for three weeks resulted in significant increases in the length and depth of tracheal submucosal glands. ${ }^{1}$ Increased cell size, acinar diameter, and lumen diameter of glands were reported in rats exposed to tobacco smoke for six weeks. ${ }^{2}$ Exposure of rats to tobacco smoke for $\mathbf{3 0}$ days increased the relative proportion of the tracheal wall occupied by glands, and also increased their mucin content. ${ }^{3}$ These animal studies have been used as models for chronic bronchitis, which is characterised primarily by hypersecretion of mucus ${ }^{4}$ and has been linked with tobacco smoking. ${ }^{5}$

Ultrastructural studies of tobacco smoke-induced mucus hypersecretion in animal models have not been traced. The present study therefore investigated the ultrastructural intracellular changes in submucosal glands from rats exposed to tobacco smoke for up to two years.

\section{Methods}

One hundred and ten male and 110 female CFHB Wistar, specific pathogen-free rats (Anglia Laboratory Animals, Huntingdon) were exposed to $6 \% \mathrm{v} / \mathrm{v}$ fresh tobacco smoke for two 20-minute periods per day, for up to two years. The rats were exposed to smoke from a conventional tobacco cigarette with a $12 \mathrm{mg}$ tar yield, in HRC rodent smoking machines. An equal number of rats was exposed to fresh air, as

Address for reprint requests: Dr DJ Lewis, Department of Pathology, Huntingdon Research Centre, Huntingdon, Cambridgeshire PE18 6ES. controls. The experiment was performed under strict barrier conditions.

All the surviving male rats in the tobacco smoke group were killed after 20 months because of a high smoke-induced mortality. Samples of trachea from $\bar{\partial}$ all rats were fixed in $10 \%$ neutral buffered formalin, $\frac{\mathscr{Q}}{\Phi}$ embedded in paraffin wax, and stained with haema- $\varnothing$ toxylin and eosin. In addition, sections from five $\overrightarrow{\overrightarrow{0}}$ male and five female rats per group were stained by 3 the high iron diamine technique. ${ }^{6}$ Ultrastructural investigations were limited to five male and five female rats from both the tobacco smoke exposed $\overrightarrow{7}$ and control groups killed after 20 months, and five 응 females from each group after two years' exposure. $\times$ All rats were killed after overnight recovery from the last smoke exposure.

The rats were killed by an intraperitoneal injection of sodium pentobarbitone. Samples of trachea $(1 \mathrm{~mm}$ rings) were fixed by immersion in $4 \%$ glutaraldehyde $\frac{\circ}{3}$ in $0.15 \mathrm{M}$ cacodylate buffer, $\mathrm{pH} 7 \cdot 3$, at $4^{\circ} \mathrm{C}$, for two hours. After washing in buffer the rings were postfixed in $1 \%$ osmium tetroxide, dehydrated in $\tilde{\sigma}$ alcohol, and embedded in epon. One micron survey sections were stained with toluidine blue and silver/ 0 gold ultrathin sections, of areas containing sub- $\omega$ mucosal glands, were stained with uranyl acetate and? lead citrate. The sections were examined with ace Philips EM 300 at $60 \mathrm{kV}$.

Before investigation of possible smoke-induced $\stackrel{\oplus}{+}$ changes, all rats were screened by light microscopy $\frac{0}{0}$ for the presence of chronic respiratory disease to $\frac{0}{\mathbb{D}}$ ensure their suitability for assessment.

\section{Results}

By light microscopy the tracheal submucosal glandso 
from smoke-exposed rats often appeared larger. In sections stained by the high iron diamine technique there were increased amounts of sulphated mucus in smoke-exposed rats.

When compared with submucosal glands from control animals, the mucous cells from smokeexposed rats illustrated marked ultrastructural changes. The most prominent change was the presence of grossly dilated cisternae of the rough endoplasmic reticulum (RER), which were filled with an amorphous electron-lucent mucus-like material (fig 1). These dilated cisternae were usually

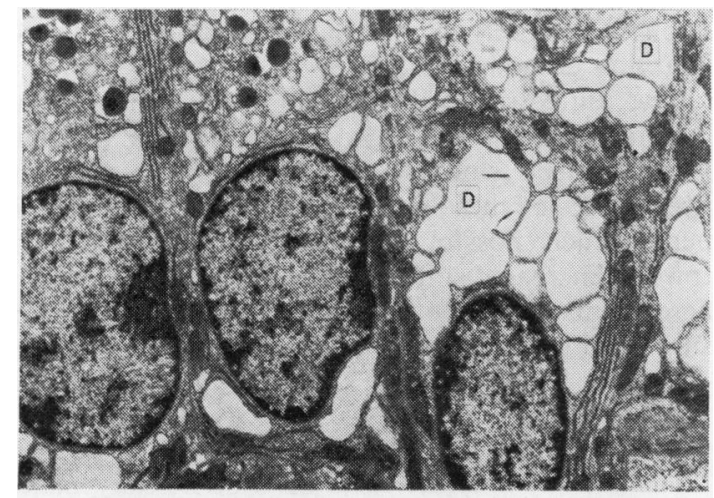

Fig 1 Mucous cell with dilated rough endoplasmic reticulum $(D)$. Original magnification $\times 10000$.

located in the basal region of the cell, but occasionally examples were seen in the supranuclear region. Not all RER cisternae were dilated; even in severely affected cells large numbers of parallel cisternae were often present (fig 2).

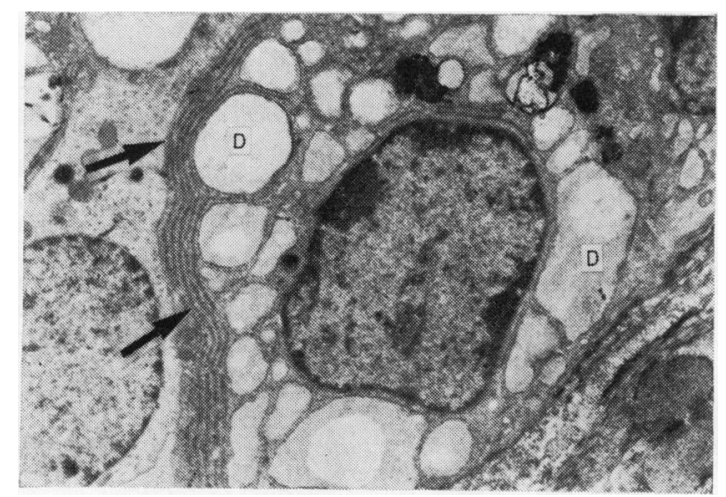

Fig 2 Mucous cell with grossly dilated rough endoplasmic reticulum (D) containing amorphous mucus-like material. Note adjacent non-dilated reticulum (arrows). Original magnification $\times 11800$.
The apical cytoplasm of the mucous cells projected bulbously into the acinar lumina and usually contained numerous secretion granules. Many of the secretion granules appeared to have coalesced and contained especially prominent electron-dense cores. In some cases when the core was large the granules resembled those found in serous cells (fig 3). The Golgi complexes of the cells appeared enlarged, with numerous vesicles and vacuoles, and occupied a large proportion of the supranuclear cytoplasm (fig 3). All other organelles within the mucous cells appeared normal and unaffected by tobacco smoke exposure.

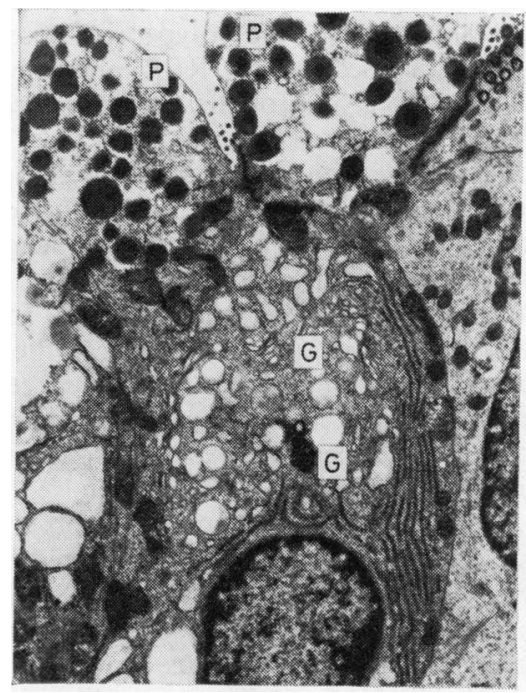

Fig 3 Mucous cells with bulbous apical projections $(P)$ filled with secretion granules containing prominent electron-dense cores. Extensive supranuclear Golgi complex $(G)$ with dilated vesicles. Original magnification $\times 10000$.

Serous, myoepithelial, and ciliated cells of the acini and ducts appeared unaffected by tobacco smoke exposure and were indistinguishable from those of control rats.

\section{Discussion}

Tobacco smoke exposure is known to produce hypertrophy of, and hypersecretion by, the submucosal glands. $^{3} 7$ Coles found that exposure of rats to tobacco smoke for six weeks caused an increase, both in size of glands (hypertrophy of the mucous tubules) and in the secretory rate of mucous cells.? He considered it likely that the rate of glycoprotein 
synthesis increased to match the higher discharge rate of mucous cells.

The results of the present study indicate that these changes are associated with ultrastructural manifestations of hyperactivity within the mucous cells. Using ultrastructural autoradiography Meyrick and Reid $^{8}$ investigated the pathway by which mucus is produced, and established the roles of individual cellular organelles. They showed that ${ }^{3} \mathrm{H}$-threonine was initially localised over the rough endoplasmic reticulum (RER) in mucous cells and later migrated to the Golgi complex. In comparison, metabolites of ${ }^{3} \mathrm{H}$-glucose were apparently primarily added in the Golgi complex. From the Golgi complex both markers were transferred into vacuoles and finally to secretion granules. Meyrick and Reid ${ }^{8}$ concluded from these findings that the polypeptide core of the mucus is synthesised in the RER and the oligosaccharides of the glycoprotein were incorporated by the Golgi complex.

Several possible sites of action for compounds which alter the rate of mucus secretion, its composition, or its rate of synthesis have been suggested. ${ }^{9}$ These sites include the genome responsible for the information for mucus synthesis; the RER in which primary polypeptide synthesis occurs; the Golgi complex; the secretory apparatus, which regulates the release of mucus from the cells; the lysosomes, which are able to degrade excess mucus; and the mucus itself, by mucolysis.

Thus, the ultrastructural changes observed in mucous cells of the present study occurred in those organelles which have been shown to be involved in mucus synthesis, ${ }^{8}$ and have also been considered possible sites at which exogenous compounds could affect mucus production. ${ }^{9}$ The gross dilation of the RER suggests that the amount of polypeptide core of the mucus within the cell was increased considerably, and was stored at the site of synthesis.

Dilatation of RER with an unusual amount of polypeptide did not provoke a lysosomal response which would be expected to occur to remove excess mucus from the cytoplasm. This suggests that the RER involved is not damaged; if it were, autophagic digestion by lysosomes would occur. Thus, the dilatation of the RER is probably a physiological cellular event representing hyperactivity, rather than a degenerative one. Similar dilatation of the RER as an intracellular storage site for cell products is known to occur in plasma cells and fibroblasts; and Ghadially suggested that there may be a defect in the transport of secretory material in these cases. ${ }^{10}$ In addition to hyperactivity of RER, hypertrophy of the Golgi complex (illustrated by increases in the extent and in the number of stacks, vacuoles, and vesicles) is usually accompanied by an increased secretory activity.

As the rats were killed after overnight recovery is from smoke exposure, the synthesis and build-up of $\overrightarrow{0}$ polypeptides within the RER is probably a result of discharge of secretion granules during exposure. $\vec{\omega}$ This stimulation of mucus secretion during irritation would be expected to stimulate rapid mucus synthesis $\vec{x}$ to replace the released granules. However, in the absence of further irritation this would lead to a build-up within the cytoplasm. Mucous cells are known alternately to accumulate and discharge their $N$ secretion granules. ${ }^{7}$

The ultrastructural appearance of the mucus $\vec{\longrightarrow}$ secretion granules was also altered in mucous cells from tobacco smoke-exposed rats. The electron- $\frac{C}{-2}$ dense cores of these granules were especially prominent, which possibly correlates with the change $\vec{\oplus}$ towards increased sulphated mucus. This finding is in $\stackrel{\infty}{-}$ agreement with the reported increased ratio of $\square$ sulphated to sialic acid mucus in human smokers. ${ }^{5}$

\section{References}

${ }^{1}$ Lamb D. Mucus secretion in hypersecretory states. Bronches 1969;18:453-65.

2 Jones R, Bolduc P, Reid L. Goblet cell glycoprotein and tracheal gland hypertrophy in rat airways: the effect of $\vec{P}$ tobacco smoke with or without the anti-inflammatory agent phenylmethyloxadiazole. Br J Exp Pathol 1973;54: 229-39.

${ }^{3}$ Hayashi M, Sornberger GC, Huber GL. Morphometric 음 analyses of tracheal gland secretion and hypertrophy in $\underset{x}{\mathscr{N}}$ male and female rats after experimental exposure to $\bar{\sigma}$ tobacco smoke. Am Rev Respir Dis 1979;119:67-73.

${ }^{4}$ Reid L. Pathology of chronic bronchitis. Lancet 1954;1: 275-9.

${ }^{5}$ Kollerstrom N, Lord PW, Whimster WF. A difference in the composition of bronchial mucus between smokers $ᄋ$ and non-smokers. Thorax 1977;32:155-9.

${ }^{6}$ Spicer SS. Diamine methods for differentiating muco- $\frac{7}{O}$ substances histochemically.J Histochem Cytochem 1965; 13:211-34.

7 Coles S. Regulation of the secretory cycles of mucous and serous cells in the human bronchial gland. In: Elstein M,N Parke DV. Mucus in health and disease. New York and London: Plenum, 1977:155-68.

${ }^{8}$ Meyrick B, Reid L. In vitro incorporation of $\left[{ }^{3} \mathrm{H}\right]$ threonine and $\left[{ }^{3} \mathrm{H}\right]$ glucose by the mucous and serous cells of the human bronchial submucosal gland. J Cell Biol 1975:67: 320-44.

9 Parke DV. Pharmacology of mucus. Br Med Bull 1978;34:89-94.

10 Ghadially FN. Ultrastructural pathology of the cell. London and Boston: Butterworths, 1975. 